\title{
The Compensation Method of Vehicle Magnetic Interference for the Magnetic Gradiometer
}

\author{
Junwei Lv, ${ }^{1}$ Zhentao Yu, ${ }^{1}$ Jingli Huang, ${ }^{1}$ and Jing Zhou ${ }^{2}$ \\ ${ }^{1}$ Department of Control Engineering, Naval Aeronautical and Astronautical University, Yantai 264001, China \\ ${ }^{2}$ Naval Unit 92474, Sanya 572098, China \\ Correspondence should be addressed to Zhentao Yu; hjhyyzt@163.com
}

Received 10 October 2013; Accepted 22 December 2013

Academic Editor: Shao-Ming Fei

Copyright (c) 2013 Junwei Lv et al. This is an open access article distributed under the Creative Commons Attribution License, which permits unrestricted use, distribution, and reproduction in any medium, provided the original work is properly cited.

\begin{abstract}
The magnetic interference of vehicle imposes a strong influence on the magnetic gradiometer. Based on the mechanism of the vehicle magnetic interference, we firstly use the difference algorithm of the magnetic gradient tensor to fuse the magnetic interference of each vector magnetometer and establish a mathematical model of vehicle magnetic interference for the magnetic gradiometer. Next, we propose a compensation method for the vehicle magnetic interference and a recognition method for the estimation of compensation coefficients based on this mathematical model. The simulation results show that the proposed method can compensate as much as $96.2 \%$ of the vehicle magnetic interference efficiently.
\end{abstract}

\section{Introduction}

The magnetic gradiometer onboard Unmanned Underwater Vehicle (UUV) for underwater object detection is attracting particular interest in recent years for its advantages in remote control and autonomous operation. The magnetic gradiometer is constructed by three spatial derivatives along orthogonal directions and a magnetic gradient is measured as the difference between two magnetic readings at different locations. The magnetic gradiometer formed by fluxgate magnetometers has several advantages such as small size and low power with relatively high sensitivity. Gradiometer has also the advantage of rejecting unwanted long-range environmental noise sources. Various critical techniques have been developed and demonstrated [1-3].

It is inevitable that the magnetic gradiometer is influenced by the magnetic interference of vehicle, which mainly includes the induced magnetic field and the permanent magnetic field of vehicle [4-7]. So it is necessary to compensate this interference. Bono et al. studied on magnetic interference of the magnetic gradiometer onboard the UUV and proposed a scheme for the compensation of vehicle magnetic interference [8]. Keene et al. developed a Superconductivity Quantum Interference Device (SQUID) gradiometer system that used active shielding and an adaptive signal processing algorithm to eliminate magnetic interference [9], but he did not expound the compensation algorithm of vehicle magnetic interference. Pei and Yeo developed a prototype magnetic gradiometer onboard UUV for magnetic detection of underwater objects, which was housed in a plastic nose cone consisting of four fluxgate magnetometers, and one of the magnetometers served as the reference reading to the earth magnetic fields and was used to compensate the platform magnetic interference of other magnetometers $[10,11]$. However this method did not consider the platform interference of the reference magnetometer.

The objective of this paper is to compensate vehicle magnetic interference for the magnetic gradiometer; we firstly propose a mathematical model of vehicle magnetic interference by using the difference algorithm of the magnetic gradient tensor to fuse the magnetic interference of each vector magnetometer and then we propose a compensation method for the vehicle magnetic interference and a recognition method for the estimation of the compensation coefficients. 
This method uses one of the magnetometers to serve as the reference and take into consideration the vehicle magnetic interference, so it can compensate the vehicle magnetic interference of the magnetic gradiometer theoretically. Finally, numerical simulations are carried out to show the effectiveness of the proposed compensation method.

\section{Preliminaries of Magnetic Gradient Tensor}

The magnetic gradient tensor is space variation rate in the $X$, $Y, Z$ directions of total magnetic field $\mathbf{B}\left(B_{x}, B_{y}, B_{z}\right)$, which is defined as

$$
\mathbf{G}=\nabla \mathbf{B}=\left[\begin{array}{ccc}
\frac{\partial B_{x}}{\partial x} & \frac{\partial B_{x}}{\partial y} & \frac{\partial B_{x}}{\partial z} \\
\frac{\partial B_{y}}{\partial x} & \frac{\partial B_{y}}{\partial y} & \frac{\partial B_{y}}{\partial z} \\
\frac{\partial B_{z}}{\partial x} & \frac{\partial B_{z}}{\partial y} & \frac{\partial B_{z}}{\partial z}
\end{array}\right]
$$

In general, the curl and the divergence of the magnetic field vanish outside the magnetic sources. So the relations of the nine gradients are expressed as

$$
\begin{gathered}
\nabla \cdot \mathbf{B}=\frac{\partial B_{x}}{\partial x}+\frac{\partial B_{y}}{\partial y}+\frac{\partial B_{z}}{\partial z}=0 \\
\nabla \times \mathbf{B}=\left|\begin{array}{ccc}
i & j & k \\
\frac{\partial}{\partial x} & \frac{\partial}{\partial y} & \frac{\partial}{\partial z} \\
B_{x} & B_{y} & B_{z}
\end{array}\right|=0 .
\end{gathered}
$$

The magnetic gradiometer is composed of four vector magnetometers and their observations are $\mathbf{B}_{i}\left(B_{x i}, B_{y i}, B_{z i}\right)$ $(i=1,2,3,4)$, which is described by

$$
\left[\begin{array}{l}
B_{x 21} \\
B_{x 31} \\
B_{x 41}
\end{array}\right]=\left[\begin{array}{lll}
d x_{21} & d y_{21} & d z_{21} \\
d x_{31} & d y_{31} & d z_{31} \\
d x_{41} & d y_{41} & d z_{41}
\end{array}\right]\left[\begin{array}{c}
\frac{\partial B_{x}}{\partial x} \\
\frac{\partial B_{x}}{\partial y} \\
\frac{\partial B_{x}}{\partial z}
\end{array}\right]
$$

where $B_{x i 1}$ is the difference between the $x$ components of the $i$ th and the first magnetometer and $\left(\begin{array}{lll}d x_{i 1} & d y_{i 1} & d z_{i 1}\end{array}\right)$ is the distance between the $i$ th and the first magnetometer [12].

Suppose $\Delta \mathbf{B}=\left(\begin{array}{lll}\mathbf{B}_{21} & \mathbf{B}_{31} & \mathbf{B}_{41}\end{array}\right), \mathbf{B}_{i 1}=\mathbf{B}_{i}-\mathbf{B}_{1}$. From (3), we derive

$$
\Delta \mathbf{B}^{T}=\mathbf{A G}^{T}
$$

where the matrix $\mathbf{A}$ is the distance matrix of (3). The magnetic gradient tensor $\mathbf{G}$ is expressed as

$$
\mathbf{G}=\Delta \mathbf{B}\left(\mathbf{A}^{T}\right)^{-1}=\Delta \mathbf{B} \cdot \mathbf{a} .
$$

Equation (5) is the difference algorithm of the magnetic gradient tensor.

\section{Mathematical Model of Vehicle Magnetic Interference}

In the process of vehicle movement, the material of vehicle can generate much magnetic interference, such as permanent field and induced field, which can impose influence on the measurement accuracy of magnetometer. The permanent field of vehicle is generated by the magnetization of the hard magnetic material. Because the hard magnetic material has high coercivity, the permanent field can be kept for a long time. Since the magnetometer and the hard magnetic material are fixedly connected on the vehicle, no matter how the vehicle posture changes, the vector magnetometer measurement of the permanent magnetic field is a constant. The induced magnetic field is generated by the magnetization of the soft magnetic material of the vehicle. The soft magnetic material has low coercivity, so the induced magnetic field changes with the external magnetic field $[13,14]$. The magnitude and direction of the induced magnetic field are associated with the posture and position of the vehicle. Three axes of the vector magnetometer are denoted as $x$-, $y$-, and $z$-axes of the coordinate system of vehicle. The permanent magnetic field of vehicle can be shown as $\mathbf{B}^{h}=\left(\begin{array}{lll}B_{x}^{h} & B_{y}^{h} & B_{z}^{h}\end{array}\right)$. Based on the Poisson equation, the induced magnetic field of vehicle $\mathbf{B}^{s}=$ $\left(\begin{array}{lll}B_{x}^{s} & B_{y}^{s} & B_{z}^{s}\end{array}\right)$ is expressed as

$$
\mathbf{B}^{s}=\mathbf{K B}^{b}
$$

where $\mathbf{B}^{b}=\left(\begin{array}{lll}B_{x}^{b} & B_{y}^{b} & B_{z}^{b}\end{array}\right)$ is the background field, $\mathbf{K}_{3 \times 3}$ is the induced coefficient matrix of the vehicle, and $\mathbf{K}_{i j}=\mathbf{K}_{j i}(i, j=$ $1,2,3)$.

By analyzing the permanent and induced magnetic field, the observation of the vector magnetometer is expressed as

$$
\mathbf{B}^{\prime}=\mathbf{B}+\mathbf{K B}^{b}+\mathbf{B}^{h}
$$

where $\mathbf{B}$ is the actual magnetic field. The observation of the $i$ th vector magnetometer of the magnetic gradiometer can be obtained from (7):

$$
\mathbf{B}_{i}^{\prime}=\mathbf{B}_{i}+\mathbf{K}_{i} \mathbf{B}^{b}+\mathbf{B}_{i}^{h},
$$

where $\mathbf{K}_{i}$ is the induced coefficient matrix of the $i$ th vector magnetometer and $\mathbf{B}_{i}^{h}$ is the permanent magnetic field of the $i$ th vector magnetometer. So the difference between the observation of the $i$ th and the first magnetometer is expressed as

$$
\mathbf{B}_{i 1}^{\prime}=\mathbf{B}_{i}^{\prime}-\mathbf{B}_{1}^{\prime}=\mathbf{B}_{i 1}+\mathbf{K}_{i 1} \mathbf{B}^{b}+\mathbf{B}_{i 1}^{h}
$$

where

$$
\begin{aligned}
& \mathbf{K}_{i 1}=\mathbf{K}_{i}-\mathbf{K}_{1}, \\
& \mathbf{B}_{i 1}^{h}=\mathbf{B}_{i}^{h}-\mathbf{B}_{1}^{h} .
\end{aligned}
$$


Based on the difference algorithm of the magnetic gradient tensor (5), the observation of the magnetic gradiometer $\mathbf{G}_{m}$ is expressed as

$$
\begin{aligned}
& \mathbf{G}_{m}=\left(\begin{array}{lll}
\mathbf{B}_{21}^{\prime} & \mathbf{B}_{31}^{\prime} & \mathbf{B}_{41}^{\prime}
\end{array}\right) \cdot \mathbf{a} \\
& =\left(\begin{array}{lll}
\mathbf{B}_{21} & \mathbf{B}_{31} & \mathbf{B}_{41}
\end{array}\right) \cdot \mathbf{a}+\left(\begin{array}{lll}
\mathbf{K}_{21} \mathbf{B}^{b} & \mathbf{K}_{31} \mathbf{B}^{b} & \mathbf{K}_{41} \mathbf{B}^{b}
\end{array}\right) \cdot \mathbf{a} \\
& +\left(\begin{array}{lll}
\mathbf{B}_{21}^{h} & \mathbf{B}_{31}^{h} & \mathbf{B}_{41}^{h}
\end{array}\right) \cdot \mathbf{a} \\
& =\mathbf{G}+\left(\begin{array}{lll}
\mathbf{K}_{21} \mathbf{B}^{b} & \mathbf{K}_{31} \mathbf{B}^{b} & \mathbf{K}_{41} \mathbf{B}^{b}
\end{array}\right) \cdot \mathbf{a} \\
& +\left(\begin{array}{lll}
\mathbf{B}_{21}^{h} & \mathbf{B}_{31}^{h} & \mathbf{B}_{41}^{h}
\end{array}\right) \cdot \mathbf{a},
\end{aligned}
$$

where $\mathbf{G}$ is the actual data of the magnetic gradient tensor. And the vehicle magnetic interference $\mathbf{D}$ is expressed as

$$
\begin{aligned}
\mathbf{D}=\mathbf{G}_{m}-\mathbf{G}= & \left(\begin{array}{llll}
\mathbf{K}_{21} \mathbf{B}^{b} & \mathbf{K}_{31} \mathbf{B}^{b} & \mathbf{K}_{41} \mathbf{B}^{b}
\end{array}\right) \cdot \mathbf{a} \\
& +\left(\begin{array}{lll}
\mathbf{B}_{21}^{h} & \mathbf{B}_{31}^{h} & \mathbf{B}_{41}^{h}
\end{array}\right) \cdot \mathbf{a} .
\end{aligned}
$$

Equation (12) is the mathematical model of the vehicle magnetic interference.

\section{Compensation Method of Vehicle Magnetic Interference}

One of the magnetometers with the maximum distance from the UUV magnetic interference can serve as the reference reading to the background field $\mathbf{B}^{b}[10,11]$. But the reference magnetometer is also influenced by vehicle magnetic interference. We take into consideration the interference of the reference magnetometer, and the background field $\mathbf{B}^{b}$ can be expressed by the actual magnetic field of the reference:

$$
\mathbf{B}^{b}=\mathbf{B}_{1}=\left(\mathbf{I}_{3 \times 3}+\mathbf{K}_{1}\right)^{-1}\left(\mathbf{B}_{1}^{\prime}-\mathbf{B}_{1}^{h}\right) .
$$

The first magnetometer serves as the reference in (13). Substituting (13) into (9), $\mathbf{B}_{i 1}^{\prime}$ can be expressed as

$$
\begin{aligned}
\mathbf{B}_{i 1}^{\prime}= & \mathbf{B}_{i 1}+\mathbf{K}_{i 1}\left(\mathbf{I}_{3 \times 3}+\mathbf{K}_{1}\right)^{-1} \mathbf{B}_{1}^{\prime} \\
& -\mathbf{K}_{i 1}\left(\mathbf{I}_{3 \times 3}+\mathbf{K}_{1}\right)^{-1} \mathbf{B}_{1}^{h}+\mathbf{B}_{i 1}^{h} \\
= & \mathbf{B}_{i 1}+\mathbf{k}_{i 1} \mathbf{B}_{1}^{\prime}+\mathbf{b}_{i 1}^{h}, \\
& \mathbf{k}_{i 1}=\mathbf{K}_{i 1}\left(\mathbf{I}_{3 \times 3}+\mathbf{K}_{1}\right)^{-1}, \\
\mathbf{b}_{i 1}^{h}= & \mathbf{B}_{i 1}^{h}-\mathbf{K}_{i 1}\left(\mathbf{I}_{3 \times 3}+\mathbf{K}_{1}\right)^{-1} \mathbf{B}_{1}^{h},
\end{aligned}
$$

where $\mathbf{k}_{i 1}$ and $\mathbf{b}_{i 1}^{h}$ are constant matrices. The observation of the magnetic gradient tensor can be expressed as

$$
\begin{aligned}
\mathbf{G}_{m}= & \left(\begin{array}{lll}
\mathbf{B}_{21}^{\prime} & \mathbf{B}_{31}^{\prime} & \mathbf{B}_{41}^{\prime}
\end{array}\right) \cdot \mathbf{a} \\
= & \left(\begin{array}{llll}
\mathbf{B}_{21} & \mathbf{B}_{31} & \mathbf{B}_{41}
\end{array}\right) \cdot \mathbf{a}+\left(\begin{array}{lll}
\mathbf{k}_{21} \mathbf{B}_{1}^{\prime} & \mathbf{k}_{31} \mathbf{B}_{1}^{\prime} & \mathbf{k}_{41} \mathbf{B}_{1}^{\prime}
\end{array}\right) \cdot \mathbf{a} \\
& +\left(\begin{array}{lll}
\mathbf{b}_{21}^{h} & \mathbf{b}_{31}^{h} & \mathbf{b}_{41}^{h}
\end{array}\right) \cdot \mathbf{a} \\
= & \mathbf{G}+\left(\begin{array}{lll}
\mathbf{k}_{21} \mathbf{B}_{1}^{\prime} & \mathbf{k}_{31} \mathbf{B}_{1}^{\prime} & \mathbf{k}_{41} \mathbf{B}_{1}^{\prime}
\end{array}\right) \cdot \mathbf{a} \\
& +\left(\begin{array}{lll}
\mathbf{b}_{21}^{h} & \mathbf{b}_{31}^{h} & \mathbf{b}_{41}^{h}
\end{array}\right) \cdot \mathbf{a} \\
= & \mathbf{G}+\mathbf{k B}_{1 m} \cdot \mathbf{a}+\mathbf{b} \cdot \mathbf{a},
\end{aligned}
$$

where $\mathbf{k}, \mathbf{B}_{1 m}$, and $\mathbf{b}$ can be expressed as

$$
\begin{aligned}
\mathbf{k} & =\left(\begin{array}{lll}
\mathbf{k}_{21} & \mathbf{k}_{31} & \mathbf{k}_{41}
\end{array}\right)_{3 \times 9}, \\
\mathbf{B}_{1 m}= & {\left[\begin{array}{ccc}
\mathbf{B}_{1}^{\prime} & \mathbf{O}_{3 \times 1} & \mathbf{O}_{3 \times 1} \\
\mathbf{O}_{3 \times 1} & \mathbf{B}_{1}^{\prime} & \mathbf{O}_{3 \times 1} \\
\mathbf{O}_{3 \times 1} & \mathbf{O}_{3 \times 1} & \mathbf{B}_{1}^{\prime}
\end{array}\right]_{9 \times 3}, } \\
\mathbf{b} & =\left(\begin{array}{lll}
\mathbf{b}_{21}^{h} & \mathbf{b}_{31}^{h} & \mathbf{b}_{41}^{h}
\end{array}\right) .
\end{aligned}
$$

The vehicle magnetic interference $\mathbf{D}$ is the measurement error, and it can be expressed by

$$
\mathbf{D}=\mathbf{k B}_{1 m} \cdot \mathbf{a}+\mathbf{b} \cdot \mathbf{a} .
$$

The actual data of the magnetic gradient tensor can be written as

$$
\mathbf{G}=\mathbf{G}_{m}-\mathbf{D}=\mathbf{G}_{m}-\mathbf{k B}_{1 m} \cdot \mathbf{a}-\mathbf{b} \cdot \mathbf{a}
$$

Equation (18) is the compensation algorithm of the vehicle magnetic interference. $\mathbf{k}$ and $\mathbf{b}$ are compensation coefficient matrices, and $\mathbf{G}_{m}, \mathbf{B}_{1 m}$ are the output of the magnetic gradiometer. In theory, (18) can be used to compensate the vehicle magnetic interference after the estimation of compensation coefficient matrices $\mathbf{k}$ and $\mathbf{b}$.

\section{Estimation of Compensation Coefficient Matrices}

The magnetic gradient tensor $\mathbf{G}$ after the compensation of magnetic interference should satisfy (2). The relation of the components of $\mathbf{G}_{m}$ and $\mathbf{D}$ can be obtained from the following:

$$
\begin{aligned}
& G_{m 12}-D_{12}=G_{m 21}-D_{21} \\
& G_{m 13}-D_{13}=G_{m 31}-D_{31}
\end{aligned}
$$




$$
\begin{aligned}
& G_{m 23}-D_{23}=G_{m 32}-D_{32}, \\
& G_{m 11}+G_{m 22}+G_{m 33}=D_{11}+D_{22}+D_{33} .
\end{aligned}
$$

We denote

$$
\begin{aligned}
f(\mathbf{k}, \mathbf{b})= & \left(\left(G_{m 12}-G_{m 21}\right)-\left(D_{12}-D_{21}\right)\right)^{2} \\
& +\left(\left(G_{m 13}-G_{m 31}\right)-\left(D_{13}-D_{31}\right)\right)^{2} \\
& +\left(\left(G_{m 23}-G_{m 32}\right)-\left(D_{23}-D_{32}\right)\right)^{2} \\
& +\left(\left(G_{m 11}+G_{m 22}+G_{m 33}\right)-\left(D_{11}+D_{22}+D_{33}\right)\right)^{2} .
\end{aligned}
$$

The estimate of the compensation coefficient matrices $\mathbf{k}$ and $\mathbf{b}$ can be taken as the function optimization, and it can be expressed as

$$
\begin{aligned}
& \min \quad F(\mathbf{k}, \mathbf{b})=\min \sum_{i=1}^{m} f_{i}(\mathbf{k}, \mathbf{b}), \\
& \text { s.t.: } \quad k_{i j}^{s} \leq k_{i j} \leq k_{i j}^{t} \quad b_{i j}^{s} \leq b_{i j} \leq b_{i j}^{t} .
\end{aligned}
$$

The value range of the components of compensation coefficient matrices $\mathbf{k}$ and $\mathbf{b}$ of ordinary vehicles is $0 \leq k_{i j} \leq$ $0.01,0 \leq b_{i j} \leq 10$. This paper proposed Genetic Algorithms (GA) method to calculate the function optimization, and the parameters of GA are chosen as follows: group size $N=30$, the gene length of the variate $n=10$, the coping probability $p_{r}=0.9$, the crossover probability $p_{c}=0.8$, and the mutation probability $p_{m}=0.8$.

\section{Numerical Simulations}

6.1. Coefficients of Vehicle Magnetic Interference. Suppose that the vehicle carries a trirectangular tetrahedron magnetic gradiometer and the induced coefficient matrix $\mathbf{K}_{i}$ and the permanent magnetic field $\mathbf{B}_{i}^{h}$ of the vector magnetometers of magnetic gradiometer are chosen as [13]

$$
\begin{aligned}
& \mathbf{K}_{1}=\left[\begin{array}{ccc}
0.5 & 0.02 & 0.03 \\
0.02 & 0.1 & 0.005 \\
0.03 & 0.005 & 0.2
\end{array}\right], \\
& \mathbf{K}_{2}=\left[\begin{array}{ccc}
0.501 & 0.0202 & 0.0301 \\
0.0202 & 0.1 & 0.005 \\
0.0301 & 0.005 & 0.2
\end{array}\right], \\
& \mathbf{K}_{3}=\left[\begin{array}{ccc}
0.5 & 0.0202 & 0.03 \\
0.0202 & 0.101 & 0.0051 \\
0.03 & 0.0051 & 0.2
\end{array}\right], \\
& \mathbf{K}_{4}=\left[\begin{array}{ccc}
0.5 & 0.02 & 0.0303 \\
0.02 & 0.1 & 0.0051 \\
0.0303 & 0.0051 & 0.202
\end{array}\right], \\
& \mathbf{B}_{1}^{h}=\left(\begin{array}{lll}
4000 & 2500 & 1800
\end{array}\right) \mathrm{nT}, \\
& \mathbf{B}_{2}^{h}=\left(\begin{array}{lll}
4010 & 2500 & 1800
\end{array}\right) \mathrm{nT}, \\
& \mathbf{B}_{3}^{h}=\left(\begin{array}{lll}
4003 & 2492 & 1800
\end{array}\right) \mathrm{nT}, \\
& \mathbf{B}_{4}^{h}=\left(\begin{array}{lll}
4000 & 2502 & 1806
\end{array}\right) \mathrm{nT} .
\end{aligned}
$$

6.2. Simulations Results. The magnetic gradiometer is used to measure the field of magnetic target which can be considered as magnetic dipole. The process of simulations is as follows.

(1) The origin values of magnetic gradient tensor are simulated by the mathematics model of magnetic dipole.

(2) Based on the mathematics model of vehicle magnetic interference, 150 groups of $\mathbf{G}_{m i}$ and $\mathbf{B}_{1 i}^{\prime}(i=$ $1,2, \ldots, 150)$ are simulated.

(3) Based on the estimation method of compensation coefficients, the compensation coefficient matrices $\widehat{\mathbf{k}}$ and $\widehat{\mathbf{b}}$ are estimated by 50 groups of $\mathbf{G}_{m i}$ and $\mathbf{B}_{1 i}^{\prime}$ :

$$
\begin{gathered}
\widehat{\mathbf{k}}=\left[\begin{array}{ccccccccc}
0.00181 & 0.00029 & 0.00025 & 0.00001 & 0.00029 & 0 & 0.00002 & 0.00001 & 0.00038 \\
0.00029 & 0 & 0.00001 & 0.00029 & 0.00144 & 0.00017 & 0.00001 & 0 & 0.00017 \\
0.00025 & 0.00001 & 0 & 0 & 0.00017 & 0 & 0.00038 & 0.00017 & 0.00313
\end{array}\right], \\
\widehat{\mathbf{b}}=\left[\begin{array}{llll}
5.36 & 4.23 & 9.16 \\
6.31 & 7.26 & 1.35 \\
3.89 & 4.59 & 2.54
\end{array}\right] \mathrm{nT} .
\end{gathered}
$$

(4) The proposed compensation algorithm and the compensation coefficient matrices $\widehat{\mathbf{k}}$ and $\widehat{\mathbf{b}}$ are used to compensate the error of the other 100 groups $\mathbf{G}_{m i}$ and obtain the estimations $\widehat{\mathbf{G}}$. The partial results of simulations are shown in Table 1.

The statistics of the simulation results show that the proposed method can compensate $96.2 \%$ vehicle magnetic 
TABLE 1: The results of magnetic interference compensation simulations.

\begin{tabular}{lcc}
\hline$i$ th $\mathbf{G}_{m i} \mathbf{B}_{1 i}^{\prime}$ & $\begin{array}{c}\text { Before compensation } \\
\left\|\mathbf{G}_{m}-\mathbf{G}_{z}\right\|_{2}(\mathrm{nT} / \mathrm{m})\end{array}$ & $\begin{array}{c}\text { After compensation } \\
\left\|\widehat{\mathbf{G}}-\mathbf{G}_{z}\right\|_{2}(\mathrm{nT} / \mathrm{m})\end{array}$ \\
\hline 1 & 53.62 & 1.351 \\
2 & 42.65 & 2.224 \\
3 & 36.15 & 1.589 \\
4 & 61.56 & 2.062 \\
5 & 41.78 & 1.239 \\
6 & 21.48 & 1.581 \\
7 & 46.24 & 0.746 \\
8 & 19.34 & 1.382 \\
\hline
\end{tabular}

interference, so the compensation method is efficient for the magnetic gradiometer.

\section{Conclusion}

Based on the study of the mechanism of the vehicle magnetic interference, we proposed a mathematical model of vehicle magnetic interference by using the difference algorithm of the magnetic gradient tensor to fuse the magnetic interference of each vector magnetometer and proposed the compensation method of the vehicle magnetic interference and the recognition method of the compensation coefficients. This method can compensate the vehicle magnetic interference of the magnetic gradiometer theoretically. The results of simulation show that the proposed method can compensate the $96.2 \%$ vehicle magnetic interference efficiently. However, it is hard to gain high precision due to the existence of so many unknown parameters in the compensation coefficients. In future works we plan to seek for other optimization algorithms to estimate the compensation coefficients and to improve the compensation of the vehicle magnetic interference.

\section{Conflict of Interests}

The authors declare that there is no conflict of interests regarding the publication of this paper.

\section{Acknowledgments}

The authors would like to thank J. Yuan, for technical assistance, and B. L. Geng and J. F. Gai, for discussions and help with the ocean remote sensing center.

\section{References}

[1] R. Stolz, V. Zakosarenko, M. Schulz et al., "Magnetic full-tensor SQUID gradiometer system for geophysical applications," The Leading Edge, vol. 25, no. 2, pp. 178-180, 2006.

[2] T. J. Gamey, T. Starr, W. E. Doll et al., "Initial design and testing of a full-tensor airborne SQUID magnetometer for detection of unexploded ordnanee," SEG Technical Program Expanded Abstraets, vol. 23, pp. 798-801, 2004.
[3] M. N. Keene, N. J. Exon, J. S. Satchell, R. G. Humphreys, N. G. Chew, and K. Lander, "HTS SQUID magnetometers with intermediate flux transformers," IEEE Transactions on Applied Superconductivity, vol. 7, no. 2, pp. 3048-3051, 1997.

[4] G. I. Allen, G. Sulzberger, J. T. Bono, J. S. Pray, and T. R. Clem, "Initial evaluation of the new real-time tracking gradiometer designed for small unmanned underwater vehicles," in Proceedings of the MTS/IEEE OCEANS, pp. 1956-1962, Washington, DC, USA, September 2005.

[5] Z. T. Yu, J. W. Lv, and B. T. Zhang, "A method to localize magnetic target based on a seabed array of magnetometers," Journal of Wuhan University of Technology, vol. 34, no. 6, pp. 131135, 2012.

[6] W. E. Tolles and N. Y. Mineola, Magnetic Field Compensation System, 1955.

[7] A. W. Fitzgibbon, M. Pilu, and R. B. Fisher, "Direct least square fitting of ellipses," IEEE Transactions on Pattern Analysis and Machine Intelligence, vol. 21, no. 5, pp. 476-480, 1999.

[8] J. T. Bono, D. J. Overway, and W. M. Wynn, "Magnetic sensor operation onboard a UUV: Magnetic noise investigation using a total-field gradiometer," in Proceedings of the IEEE Oceans, pp. 2018-2022, San Diego, Calif, USA, September 2003.

[9] M. N. Keene, K. P. Humphrey, and T. J. Horton, "Actively shielded, adaptively balanced SQUID gradiometer system for operation aboard moving platforms," IEEE Transactions on Applied Superconductivity, vol. 15, no. 2, pp. 761-764, 2005.

[10] Y. H. Pei and H. G. Yeo, "UXO survey using vector magnetic gradiometer on autonomous underwater vehicle," in Proceedings of the MTS/IEEE Biloxi-Marine Technology for Our Future: Global and Local Challenges (OCEANS '09), Biloxi, Mississippi, October 2009.

[11] Y. H. Pei and H. G. Yeo, "Magnetic gradiometer inversion for underwater magnetic object parameters," in Proceedings of the Asia Pacific (OCEANS '06), Singapore, May 2006.

[12] K. K. Khurana, E. L. Kepko, M. G. Kivelson, and R. C. Elphic, "Accurate determination of magnetic field gradients from fourpoint vector measurements - II: use of natural constraints on vector data obtained from four spinning spacecraft," IEEE Transactions on Magnetics, vol. 32, no. 5, pp. 5193-5205, 1996.

[13] X. Zhang and Y. Zhao, "New auto-calibration and compensation method for vehicle magnetic field based on ellipse restriction," Chinese Journal of Scientific Instrument, vol. 30, no. 11, pp. 24382443, 2009.

[14] J. L. Crassidis, K.-L. Lai, and R. R. Harman, "Real-time attitudeindependent three-axis magnetometer calibration," Journal of Guidance, Control, and Dynamics, vol. 28, no. 1, pp. 115-120, 2005. 


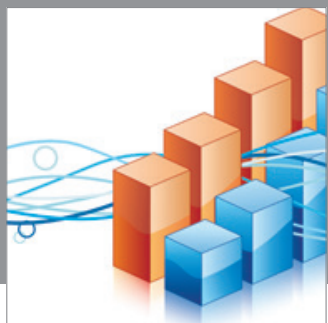

Advances in

Operations Research

mansans

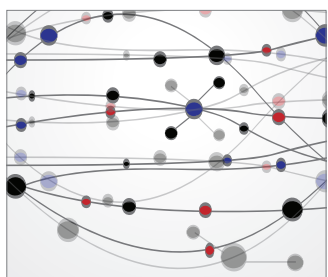

The Scientific World Journal
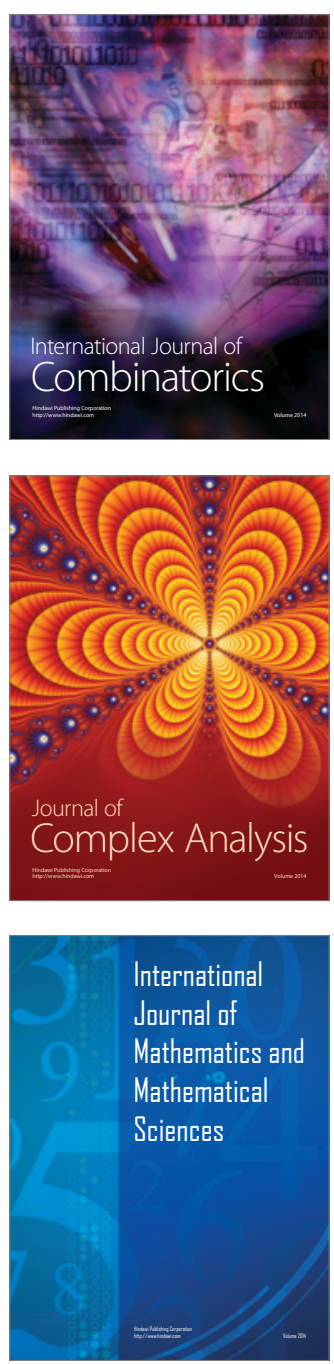
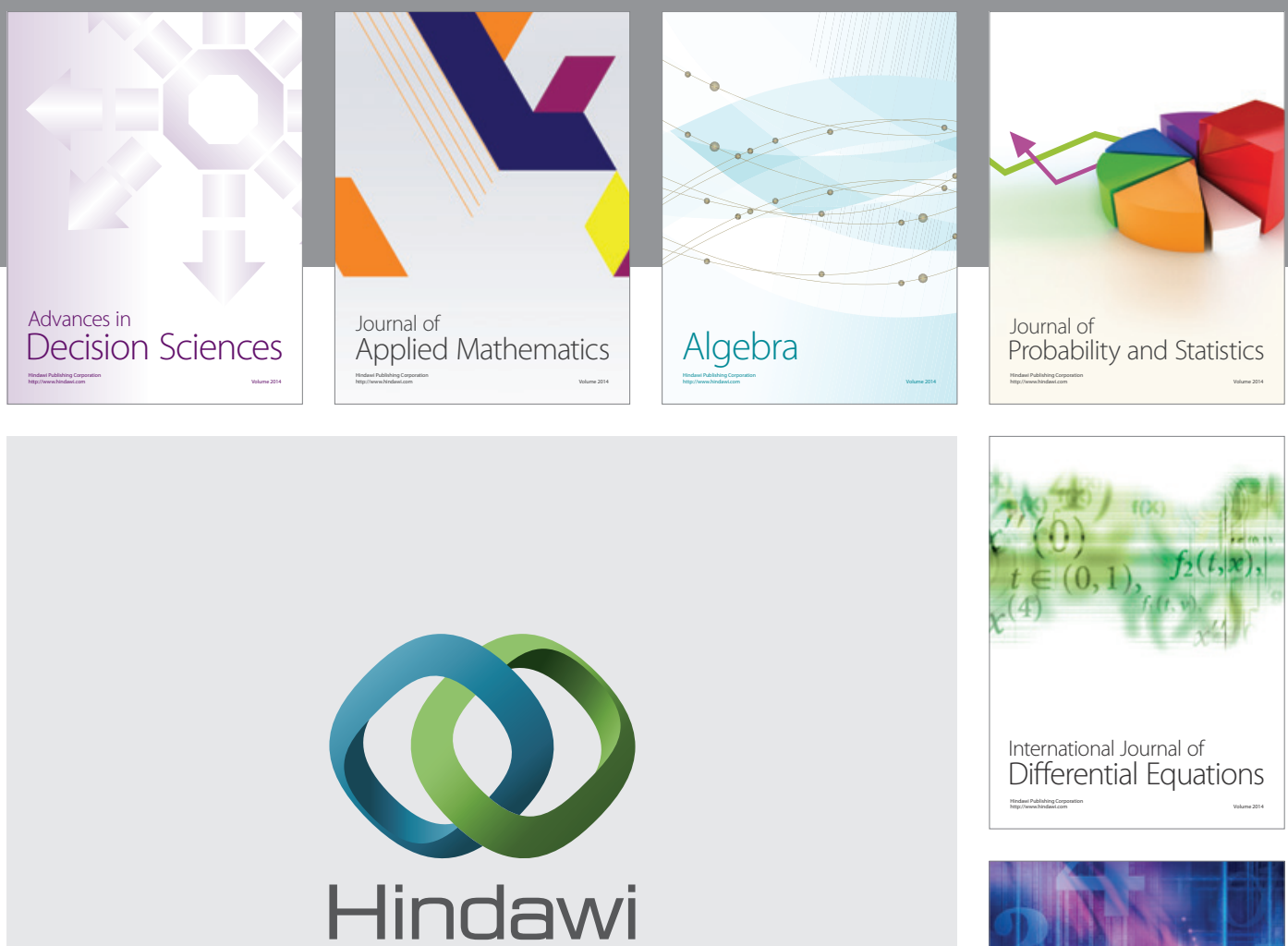

Submit your manuscripts at http://www.hindawi.com
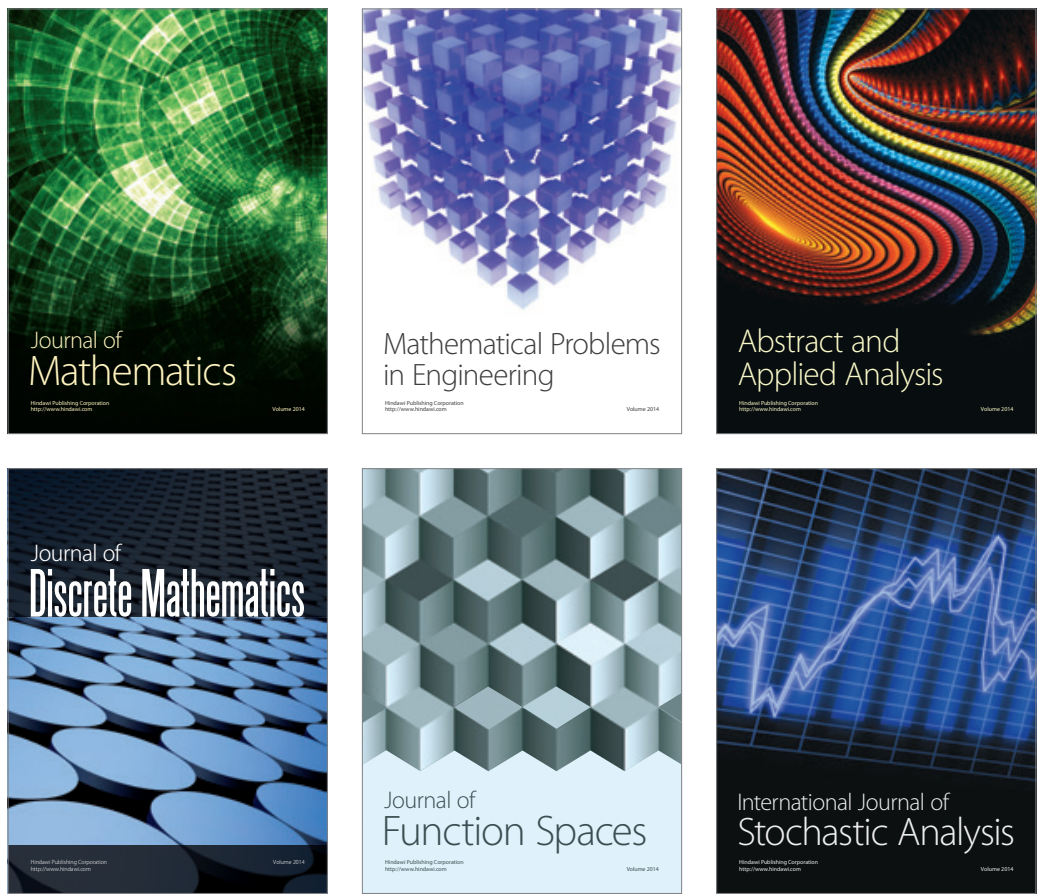

Journal of

Function Spaces

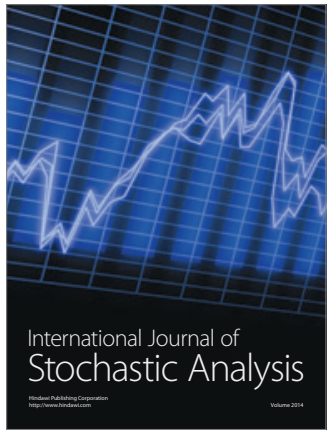

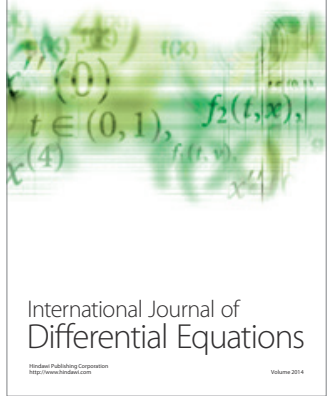
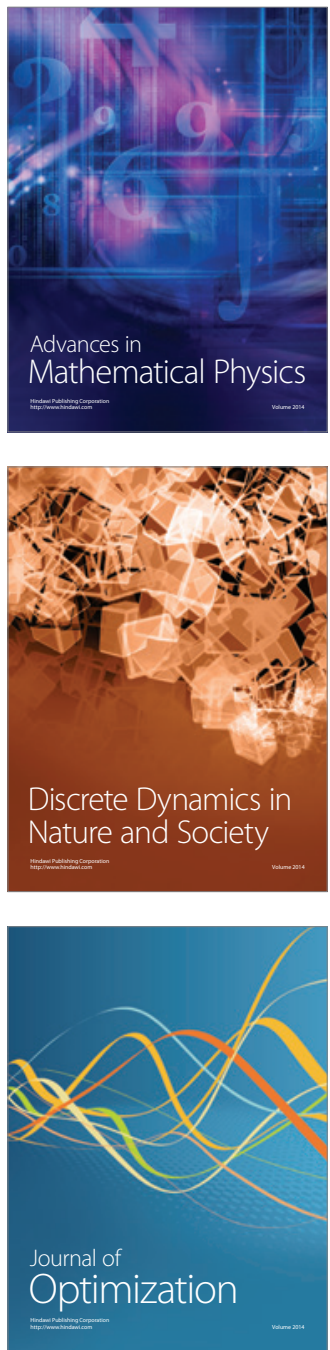